\title{
MICALs in control of the cytoskeleton, exocytosis, and cell death
}

\author{
Yeping Zhou • Rou-Afza F. Gunput • \\ Youri Adolfs $\cdot$ R. Jeroen Pasterkamp
}

Received: 21 June 2011/Revised: 14 July 2011/Accepted: 19 July 2011/Published online: 6 August 2011

(C) The Author(s) 2011. This article is published with open access at Springerlink.com

\begin{abstract}
MICALs form an evolutionary conserved family of multidomain signal transduction proteins characterized by a flavoprotein monooxygenase domain. MICALs are being implicated in the regulation of an increasing number of molecular and cellular processes including cytoskeletal dynamics and intracellular trafficking. Intriguingly, some of these effects are dependent on the MICAL monooxygenase enzyme and redox signaling, while other functions rely on other parts of the MICAL protein. Recent breakthroughs in our understanding of MICAL signaling identify the ability of MICALs to bind and directly modify the actin cytoskeleton, link MICALs to the docking and fusion of exocytotic vesicles, and uncover MICALs as anti-apoptotic proteins. These discoveries could lead to therapeutic advances in neural regeneration, cancer, and other diseases.
\end{abstract}

Keywords Apoptosis - Axon guidance - Cytoskeleton Exocytosis · NDR kinase · Plexin · Rab GTPase · Semaphorin

$\begin{array}{ll}\text { Abbreviations } & \\ \text { CC } & \text { Coiled-coil } \\ \text { Cdk5 } & \text { Cyclin-dependent kinase 5 } \\ \text { CH } & \text { Calponin homology } \\ \text { CNS } & \text { Central nervous system } \\ \text { CRMP } & \text { Collapsin response mediator protein } \\ \text { EGCG } & \text { Epigallocatechin gallate }\end{array}$

Y. Zhou · R.-A. F. Gunput · Y. Adolfs · R. J. Pasterkamp (凹) Department of Neuroscience and Pharmacology, University Medical Center Utrecht, STR 4.229, Universiteitsweg 100, 3584 CG Utrecht, The Netherlands e-mail: r.j.pasterkamp@umcutrecht.nl

$\begin{array}{ll}\text { FAD } & \text { Flavin adenine dinucleotide } \\ \text { FBD } & \text { FAD binding domain } \\ \text { GSK-3 } \beta & \text { glycogen synthase kinase-3 } \beta \\ \text { LIM } & \text { Lin11, Isl-1, Mec-3 } \\ \text { MAPRE } & \text { Microtubule-associated RP/EB } \\ \text { MICAL } & \text { Molecule interacting with CasL } \\ \text { MICAL-L } & \text { MICAL-like } \\ \text { MICAL2-PV } & \text { MICAL2 prostate cancer variant } \\ \text { MO } & \text { Flavoprotein monooxygenase } \\ \text { MST } & \text { Mammalian Ste20-related } \\ \text { NADPH } & \text { Nicotinamide adenine dinucleotide } \\ & \text { phosphate } \\ \text { NDR } & \text { Nuclear Dbf2-related } \\ \text { Npn } & \text { Neuropilin } \\ \text { PHBH } & P \text {-hydroxybenzoate hydroxylase } \\ \text { PIP2 } & \text { Phosphatidylinositol 4, 5-biphosphate } \\ \text { PlexA } & \text { Drosophila plexinA } \\ \text { RanBPM } & \text { Ran GTPase binding protein } \\ \text { RASSF1A } & \text { Ras association domain family member 1A } \\ \text { Sema } & \text { Semaphorin } \\ \text { TNF } \alpha & \text { Tumor necrosis factor } \alpha \\ \text { TRX } & \text { Thioredoxin }\end{array}$

\section{Introduction}

In 2002, the founding members of the MICAL family, human MICAL-1 and Drosophila Mical, were identified [1-3]. MICAL-1 was found in a screen for CasL-interacting proteins (hence the name MICAL for molecule interacting with CasL) [2], while a search for proteins binding to the cytoplasmic domain of plexin receptors led to the discovery of Mical [3]. Since the original identification of MICAL-1, two more MICAL proteins (MICAL-2 
and MICAL-3) have been identified in human and rodents on the basis of amino acid sequence and structural similarities (Fig. 1a) [2-6]. Eight MICAL homologues have been reported in zebrafish [7]. In addition to MICALs, a group of MICAL-like (MICAL-L) proteins has been described. MICAL-Ls have an overall domain organization similar to MICALs but they lack the conserved N-terminal region (Fig. 1b). Drosophila has one Mical-L protein, while mice and human have two, MICAL-L1 and JRAB/ MICAL-L2 [3, 8]. The focus of the present review will be on MICAL proteins. The function and mechanism-ofaction of MICAL-L proteins have been described in detail in several recent reviews [9-12].

MICALs are unusual multidomain proteins as they contain an N-terminal flavoprotein monooxygenase (MO) domain in addition to a calponin homology $(\mathrm{CH})$ domain, an LIM domain, and coiled-coil (CC) motifs linked by nonconserved variable regions (Fig. 1a). The combination of an MO domain with several different protein-protein interaction domains in one protein is unique and invites the speculation that MICALs may interact with multiple different proteins and control their activity through redox modifications [3]. MICALs function in several different physiological and pathological processes. In Drosophila, Mical is required for motor axon pathfinding, synaptic bouton distribution, and dendritic pruning during neural development [3, 13, 14]. Furthermore, Drosophila Mical influences myofilament patterning in muscles and bristle formation [13, 15]. Vertebrate MICALs have been

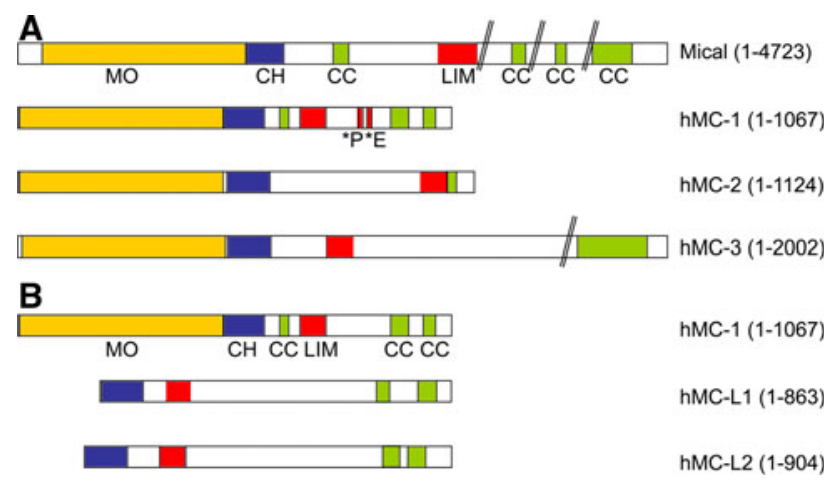

Fig. 1 MICALs form an evolutionary conserved family of signaling proteins. a Domain organization of Drosophila Mical and human MICAL-1, MICAL-2, and MICAL-3 (hMC-1, -2, and -3). MICALs contain an N-terminal flavoprotein monooxygenase domain $(\mathrm{MO}$; yellow), followed by a calponin homology $(\mathrm{CH}$; blue $)$ domain, LIM domain (red) and coiled-coil motifs $(C C$; green). These domains are linked by poorly conserved variable regions (white regions). In the hMC-1 protein, a PPKPP motif $\left({ }^{*} \mathrm{P}\right)$ and a glutamic acid-rich motif $(* \mathrm{E})$ are present. These motifs are not found in other MICALs. Numbers indicate amino acid positions. b Comparison of hMC-1, MICAL-Like1 and 2 [hMC-L1 and -L2 (JRAB)]. MICAL-Ls show a similar domain organization as MICALs but lack the N-terminal MO domain implicated in axon guidance, positioning of motor neuron cell bodies, and axon outgrowth in the developing nervous system, in exocytosis, apoptosis, and central nervous system (CNS) regeneration [2, 4-6, 16-21].

In this review, we summarize and discuss the recent progress in our understanding of MICAL signaling and function. Of the MICAL proteins, Mical and MICAL-1 have been analyzed in most detail using different expression, biochemical, and functional approaches. Therefore, the following sections focus on our current knowledge of the structural organization, regulatory mechanism, expression, and function of Mical and MICAL-1, supplemented by knowledge of other MICALs.

\section{Structure and domain organization of MICALs}

MICAL proteins have a unique structure as they combine an N-terminal enzymatic region with several protein-protein interaction modules that are known to interact with cytoskeletal and signaling cues when present in other proteins. Here we discuss the different protein domains that have been identified in MICALs (see also [11]).

\section{Flavoprotein monooxygenase domain}

Flavoprotein monooxygenases are enzymes that catalyze chemical reactions, e.g., the addition of a single oxygen atom from molecular oxygen into a substrate, via the cofactor flavin. The MICAL MO domain is located at the most N-terminal part of MICAL proteins and covers about 500 amino acids (Fig. 1a). It is conserved among family members, but is clearly distinct from previously described flavoprotein monooxygenases. Within the MICAL MO domain, three separate conserved motifs can be discerned that define the flavin adenine dinucleotide (FAD) binding domain (FBD) present in flavoprotein monooxygenases. The amino acid sequence and spacing of these three motifs resembles those found in other monooxygenases [3, 22, 23]. Together with studies on the tertiary structure of the MICAL-1 MO domain and enzymatic experiments [20-23], these features support the idea that MICALs are enzymatically active flavoprotein monooxygenases.

The overall topology of the MICAL MO domain closely resembles that of $\mathrm{PHBH}$ ( $p$-hydroxybenzoate hydroxylase), a NADPH-dependent monooxygenase [22-25]. Not surprisingly, the activity of the MICAL-1 MO domain is also NADPH-dependent [15, 20, 22]. PHBH catalyzes the oxidization of small molecules such as $p$-hydroxybenzoate and steroids. In contrast, because of the large active-site cavity in the MICAL-1 MO domain, it has been suggested that the MO domain of MICALs may have larger substrates, such 
as proteins [22, 23]. Enzymatic experiments suggest that CRMPs (collapsin response mediator proteins) and actin filaments may be substrates for the MICAL MO domain. Interestingly, F-actin binds the MO domain directly while CRMPs interact with other parts of the protein (see also "Cytoskeletal regulation") $[15,20]$.

The MICAL MO domain is required for several of the reported functions of MICAL proteins. For example, sitedirected mutagenesis of the first FAD fingerprint in the Mical MO domain leads to a loss of axon repulsion induced by semaphorins in Drosophila in vivo [3]. Similarly, transfection of a dominant negative MICAL-1 mutant lacking the N-terminal MO domain or addition of EGCG (epigallocatechin gallate), a green tea extract known to inhibit flavoprotein monooxygenases [3, 26, 27], to neuron cultures can reduce axon outgrowth inhibition and repulsion induced by the axon guidance protein Sema3A [3, 5, 20]. Finally, during the docking and fusion of secretory vesicles, MICAL-3 redox activity has been proposed to promote vesicle fusion by inducing MICAL-3 protein turnover and thereby remodeling of the vesicle-docking protein complex in which it is engaged [18].

\section{Calponin homology domain}

The MO domain of MICALs is followed by a $\mathrm{CH}$ domain (Fig. 1a). The $\mathrm{CH}$ domain was first described in calponin, an actin-binding protein with a regulatory role in muscle contractility and non-muscle cell motility [28]. Three main types of $\mathrm{CH}$ domains have been described. A combination of type 1 and $2 \mathrm{CH}$ domains forms the actin-binding domain of a large number of F-actin interacting proteins. Type $1 \mathrm{CH}$ domains have the intrinsic ability to bind to F-actin, whereas type 2 domains lack this binding ability but are required to facilitate high-affinity binding [29]. Type $2 \mathrm{CH}$ domains are present in proteins such as MAPRE (microtubule-associated RP/EB proteins) and contain a PIP2 (phosphatidylinositol 4, 5-biphosphate) binding site implicated in the regulation of F-actin [30, 31]. Type $3 \mathrm{CH}$ domains can be found as single $\mathrm{CH}$ domains in several cytoskeletal and signaling proteins [29, 32].

Structural work has shown that MICALs contain a type $2 \mathrm{CH}$ domain [33]. Purified human MICAL-1 $\mathrm{CH}$ domain does not bind F-actin [33] and the $\mathrm{CH}$ domain of Drosophila Mical is not required for F-actin interactions [15]. Thus, the MICAL CH domain may be necessary to facilitate F-actin binding to the MO domain but most likely does not bind actin directly [15]. Alternatively, however, the MICAL CH domain may function as a protein-protein interaction module for proteins other than actin [29, 32]. The tertiary structure of the MICAL-1 CH domain reveals the presence of hydrophobic residues on its surface, which may facilitate intra- or intermolecular interactions [33].
However, it remains to be determined if MICAL proteins can recruit interactors via their $\mathrm{CH}$ domain.

\section{LIM domain}

The LIM (Lin11, Isl-1, and Mec-3) domain is another domain shared by all MICAL proteins. It is connected to the $\mathrm{CH}$ domain by a variable region, which is not conserved among family members and variable in length (Fig. 1a). LIM domains are cytoskeletal interaction elements and have been implicated in mediating specific protein-protein interactions $[34,35]$. The MICAL LIM domain belongs to the monooxygenase or kinase catalytic group of LIM domains [36, 37] and seems to be a docking site for other protein(s). For example, the LIM domain of MICAL-1 mediates binding to CRMP-1 and NDR (nuclear Dbf2-related) kinases [20, 21]. Interestingly, the MICAL-1 LIM domain also mediates an intramolecular interaction with the C-terminal region that leads to an autoinhibited protein conformation [20].

\section{Proline-rich regions}

Proline-rich regions are not only important for proper protein folding but also have the potential to interact with other proteins $[38,39]$. MICAL-1 contains a proline-rich region close to its C-terminal coiled-coil motifs. Within this region, a PPKPP (Pro-Pro-Lys-Pro-Pro) motif has been identified that mediates the interaction with the SH3 domain of the Cas family members Cas and CasL (Fig. 1a) [2]. The PPKPP motif is conserved between mouse and human MICAL-1, but is not present in MICAL-2 and 3 [5]. However, in all vertebrate and invertebrate MICALs, proline-rich regions can be found scattered throughout the protein sequence. It is therefore possible that other MICALs utilize proline-rich regions for interacting with other proteins.

\section{Glutamic acid-repeat}

Human and mouse MICAL-1, but not other MICAL proteins, contain a glutamic acid-repeat region in the vicinity of the C-terminal coiled-coil motifs (Fig. 1a) [5, 6]. The function of this stretch is unknown, but several proteins have been shown to interact with histones through their glutamic acid-rich regions [40-42].

Coiled-coil motifs

The coiled-coil motif is a ubiquitous protein-folding and assembly motif. Predictions based on the analysis of primary sequences suggest that approximately $2-3 \%$ of all proteins form coiled coils [43, 44]. All MICAL proteins 
possess several coiled-coil motifs (Fig. 1). In MICAL-1, coiled-coil motifs are present in the C-terminal region (Fig. 1a). This region is involved in interactions with plexinA, vimentin, Rab1, and NDRs [2, 3, 6, 20, 21]. Furthermore, in MICAL-1, this region binds to the LIM domain-containing part of the protein, thereby inducing an intramolecular interaction that autoinhibits MO domain activity [20]. It remains to be shown, however, whether the coiled-coil motifs or other sequences in the C-terminal region are required for mediating these inter- and intramolecular interactions.

In conclusion, MICAL proteins contain a number of wellknown protein-protein interaction modules with the potential to recruit other structural or signaling proteins. Although several proteins have been identified as interactors of the C-terminal region of MICALs (Table 1), binding partners for other protein domains remain largely unknown.

\section{Regulation of MICAL-1}

Deletion of the C-terminal region (the last 290 amino acids) of mouse MICAL-1 leads to a large increase in the enzymatic activity of the MICAL-1 MO domain (as monitored by hydrogen peroxide $\left(\mathrm{H}_{2} \mathrm{O}_{2}\right)$ production) and in cell contraction $[20,21]$. These effects can be inhibited by the addition of purified C-terminal region to cell lysates (for $\mathrm{H}_{2} \mathrm{O}_{2}$ production) or by expression of the C-terminal part of MICAL- 1 in cells (for cell contraction). Furthermore, the C-terminal region of MICAL-1 can physically interact with the N-terminal part of MICAL-1 containing the LIM domain. Together, these results support a model in which a physical interaction between the $\mathrm{N}$ - and C-terminal regions of
MICAL-1 induces an autoinhibitory conformation of MICAL-1 that restricts MO activity [20]. How this autoinhibitory mechanism is regulated is unknown, but it has been suggested that proteins interacting with the C-terminal region of MICALs may release the autoinhibitory state and induce enzymatic activity. For example, binding of plexinAs or Rabs to MICALs may release the autoinhibition and activate the protein. A possible mechanism through which MICALs may be activated is phosphorylation. Biochemical studies show that MICAL-1 is phosphorylated, both in cells in culture and in brain tissue in vivo [21]. Although MICAL1 cooperates with NDR $1 / 2$ and MST1 in the control of apoptosis, it does not serve as a substrate for these kinases [21]. In addition to MICAL-1, human MICAL-2 is phosphorylated on Y653 in non-small lung cancer cell lines and tumors and MICAL-3 on residues S649 and T684 [45, 46]. Therefore, an important future goal is to identify the kinases that phosphorylate MICALs and to establish the functional relevance of these phosphorylation events.

\section{Expression and distribution of MICALs}

In cell lines and neurons (our own unpublished observations), endogenous or exogenous MICAL-1 mainly localizes to the cytosol and occasionally to filopodia or lamellipodia, but is absent from the nuclear compartment $[2,20]$. This is supported by cell fractionation studies showing that endogenous MICAL-1 is present in the cytoplasmic fraction and marginally in membrane fractions [6]. MICAL-3 has also been found in the cytosol [4]. The cellular distribution of the MICAL-2 protein is currently unknown.

Table 1 MICAL interacting proteins

\begin{tabular}{|c|c|c|c|c|}
\hline MICAL & Species & Interacting protein & (Proposed) Function & Reference \\
\hline \multirow[t]{2}{*}{ Mical } & \multirow[t]{2}{*}{ Drosophila } & plexinA & Axon guidance & [3] \\
\hline & & F-actin & Cytoskeleton & [15] \\
\hline \multirow[t]{10}{*}{ MICAL-1 } & Human & Rab1, 8a, 10, 13, 15, 35, 36 & Vesicle trafficking & {$[4,6,18,60,61]$} \\
\hline & \multirow[t]{2}{*}{ Human } & CasL & Cytoskeleton & [2] \\
\hline & & Vimentin & Cytoskeleton & [2] \\
\hline & \multirow[t]{2}{*}{ Mouse } & CRMP-1 & \multirow[t]{2}{*}{ Axon guidance } & \multirow[t]{2}{*}[19,20]{} \\
\hline & & CRMP-2 & & \\
\hline & \multirow[t]{2}{*}{ Mouse } & plexinA1 & \multirow[t]{2}{*}{ Axon guidance } & \multirow[t]{2}{*}{ [20] } \\
\hline & & plexinA3 & & \\
\hline & \multirow[t]{2}{*}{ Mouse } & NDR1 & \multirow[t]{2}{*}{ Apoptosis } & \multirow[t]{2}{*}{ [21] } \\
\hline & & NDR2 & & \\
\hline & Mouse & RanBPM & Axon guidance & {$[88]$} \\
\hline MICAL-2 & Human & Rab1 & Vesicle trafficking & [4] \\
\hline \multirow[t]{2}{*}{ MICAL-3 } & \multirow[t]{2}{*}{ Human } & Rab1, 8a, 35 & \multirow[t]{2}{*}{ Vesicle trafficking, fusion, and docking } & \multirow[t]{2}{*}[4,18,60,61]{} \\
\hline & & ELKS & & \\
\hline
\end{tabular}


MICAL proteins are expressed in a large number of different tissues. For example, in human and mice, MICAL-1 and MICAL-3 proteins are detected in tissues such as brain, lung, spleen, and kidney [2, 4]. The specific distribution of MICALs in the zebrafish and in the rodent brain have been studied in detail using Northern-blot analysis and in situ hybridization [5, 7]. In the rat brain, $M I C A L-1$ and $M I C A L-3$ transcripts are expressed at comparable levels from E15 (embryonic day 15) to adulthood [5]. MICAL-2 transcripts can only be detected starting from E18 and levels increase towards adulthood [5]. The spatiotemporal distribution patterns of the different MICALs in brain tissue are highly similar with a few exceptions. For example, MICAL-2 expression is delayed and is absent from the striatum and hypothalamus where $M I C A L-1$ and $M I C A L-3$ are found [5]. These expression patterns indicate overlapping and distinct functions for the three vertebrate MICALs during brain development and homeostasis [5].

\section{Cellular functions of MICAL proteins}

MICALs exert different cellular effects via interactions with membrane-associated or cytoplasmic proteins. The proteins currently known to interact with MICALs are discussed in light of their reported cellular functions. The role of MICALs during neural development is discussed in a separate section.

\section{Cytoskeletal regulation}

Several lines of experimental evidence implicate MICALs in F-actin regulation. Mical is required for bristle development, which is an actin-dependent process [47], downstream of Sema-1a and plexinA (PlexA) in Drosophila [15]. Decreasing or increasing Mical levels in bristles causes abnormal bristle branching and accompanying defects in actin filaments [15]. In addition, Mical deficient larva show disorganized and accumulated actin and myosin in somatic muscles [13] and defects in dendritic pruning, another actin-dependent process [14]. The Mical MO domain can bind F-actin and activated Mical (lacking the $\mathrm{C}$-terminal region of the protein) induces actin depolymerization and a reduction in actin filament length in vitro [15]. These results define an exciting novel role for the Mical MO domain and redox signaling in the control of actin dynamics. Oxidation of actin leads to the disassembly of actin filaments, reduced ability of actin to interact with actin-cross-linking proteins, and a decrease in actin polymerization [48-50]. An important point to address in future studies is whether the Mical MO domain directly modifies F-actin or uses an intermediate such as $\mathrm{H}_{2} \mathrm{O}_{2}$ to do so, as has been suggested for CRMPs [19].
In addition to F-actin, MICALs physically interact with vimentin ([2]; Y.Z. and R.J.P., unpubl. obs.). Interestingly, vimentin can be oxidized by $\mathrm{H}_{2} \mathrm{O}_{2}$ leading to cytoskeletal rearrangements [51], raising the possibility that MICALs regulate vimentin intermediate filaments through oxidation. Tubulin can also be oxidized and its oxidation abolishes polymerization activity [52]. MICAL-1 and MICAL-3 show a filamentous distribution in HeLa cells and this specific distribution is disrupted by nocodazole treatment suggesting a link to microtubules [4]. However, these data are not supported by studies in Drosophila showing that Mical does not associate directly with microtubules and that activated Mical fails to affect tubulin polymerization in vitro [15].

MICALs may also affect the cytoskeleton indirectly through their interacting proteins. For example, MICAL-1 binds Cas and CasL [2], members of the p130 Cas family, which have been implicated in the regulation of actin dynamics [53]. MICAL-1 also binds and negatively regulates NDR2 kinase, which can associate with actin in vitro $[21,58]$. Finally, another binding partner of MICAL-1 that provides an indirect link to the cytoskeleton is CRMP-2. CRMP-2 can associate with tubulin heterodimers and promotes microtubule assembly [54, 55]. MICAL-1 binds CRMP-2, and other CRMP family members [20], and is required for the oxidation-dependent phosphorylation of CRMP-2 by GSK-3 $\beta$ (glycogen synthase kinase-3 $\beta$ ) (Fig. 2a). In brief, following stimulation of neurons with Sema3A, Cdk5 (cyclin dependent kinase 5) phosphorylates CRMP-2 at Ser522, which acts as a priming event for GSK-3 $\beta$-mediated CRMP-2 phosphorylation at Thr509, Thr514, and Ser518 leading to microtubule disassembly and growth cone collapse [55-57]. Interestingly, recent work supports a model in which Sema3A stimulation also leads to the oxidation of CRMP- 2 by $\mathrm{H}_{2} \mathrm{O}_{2}$ generated by activated MICAL (Fig. 2a). Oxidized CRMP-2 forms disulfide-linked homodimers which induces the formation of a transient complex between CRMP-2 and thioredoxin (TRX). The interaction between CRMP-2 and TRX is required for CRMP-2 phosphorylation by GSK-3 $\beta$, leading to microtubule disassembly and growth cone collapse [19]. Thus, MICALs may both directly and indirectly regulate the cytoskeleton.

MICAL-3 contributes to exocytosis through Rab proteins

All three MICAL proteins (MICAL-1, MICAL-2, and MICAL-3) can interact with small GTPases of the Rab family [59]. MICAL-1, MICAL-2, and MICAL-3 bind $\mathrm{Rab} 1 \mathrm{~A} / \mathrm{B}$, involved in vesicle trafficking between the endoplasmic reticulum and the Golgi complex [4, 6]. An interaction with Rab8A/B and Rab35 has been reported for 


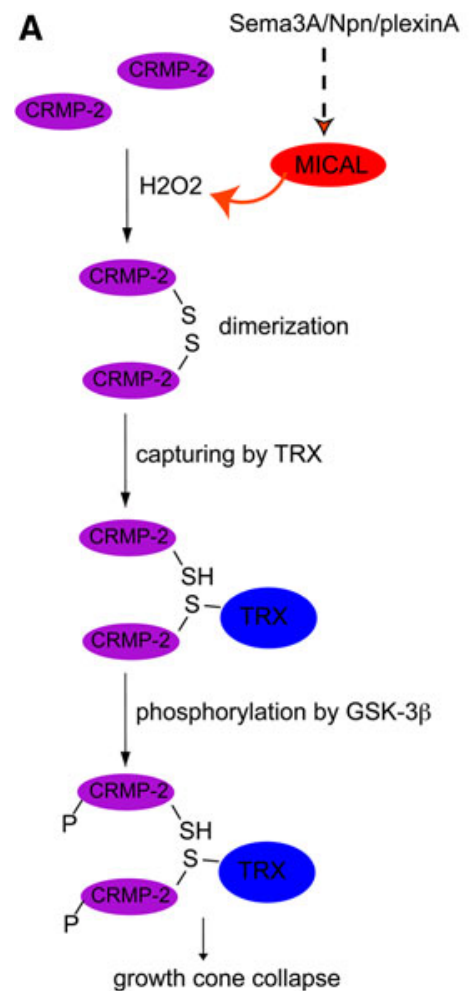

Fig. 2 Novel functions of MICAL proteins. a Role of MICAL-1 in CRMP-2 (collapsin response mediator protein 2) phosphorylation during axon guidance. Stimulation of axons with Sema3A induces $\mathrm{H}_{2} \mathrm{O}_{2}$ production by MICAL- $1 . \mathrm{H}_{2} \mathrm{O}_{2}$ oxidizes CRMP-2 to form a disulfide-linked homodimer. Oxidized CRMP-2 is then reduced by thioredoxin (TRX) and TRX forms a disulfide link with one molecule of CRMP-2. Formation of a complex between CRMP-2 and TRX promotes phosphorylation of CRMP- 2 by GSK- $3 \beta$ resulting in growth cone collapse [19]. Npn neuropilin. b Role of MICAL-3 in vesicle docking and fusion. During vesicle docking, Rab8 interacts with LL5 $\beta /$ ELKS-positive sites in the plasma membrane through MICAL-

MICAL-1 and MICAL-3, while MICAL-1 associates with Rab10, Rab13, Rab15, and Rab36 [18, 60, 61]. MICAL-Ls (MICAL-L1 and MICAL-L2) interact with a strikingly similar set of Rab proteins and cooperate with Rabs in endocytic trafficking (for a recent review, see [10-12]). A functional link between MICALs and Rabs has recently been shown for MICAL-3 and Rab8 (Fig. 2b). Rab8A associates with exocytotic vesicles in a Rab6-dependent manner and is required for vesicle docking and fusion. MICAL-3 serves as a physical link between Rab8A and ELKS, a Rab6-interacting protein expressed at the plasma membrane required for vesicle docking and fusion [18]. A functional role for MICAL-3 in the membrane fusion of Rab8-positive vesicles through ELKS is supported by the observation that expression of dominant-negative forms of MICAL-3 does not affect vesicle docking but induces a strong accumulation of secretory vesicles at the cell cortex [18]. The effect of MICAL-3 in vesicle fusion is dependent on an intact MO domain and a model has been proposed in
C
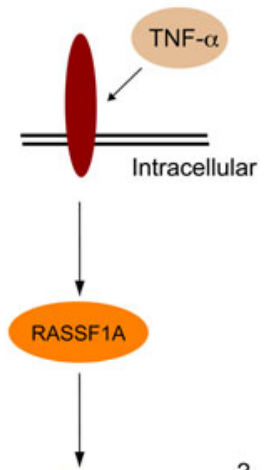

MST1

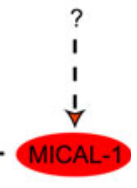

3, which binds Rab8 and ELKS. Redox activity of MICAL-3 promotes MICAL-3 turnover and remodeling of the docking complex leading to vesicle fusion [18]. c Role of MICAL-1 in NDR kinasemediated pro-apoptotic signaling. Pro-apoptotic signals such as TNF$\alpha$ induce activation of NDR1 and NDR2 kinases through the RASSF1A-MST1 pathway. MST1 phosphorylates the C-terminal hydrophobic motif of NDR kinases leading to their activation and apoptosis. MICAL-1 competes with MST1 for binding to the C-terminal part of NDRs and thereby negatively regulates proapoptotic signaling through the MST1-NDR pathway. The signals that regulate MICAL-1 in this pathway are unknown [21]

which MICAL-3 redox function controls MICAL-3 protein turnover. Rapid turnover of MICAL-3 may promote vesicle fusion by destabilizing the vesicle-docking protein complexes in which it is engaged [18] (Fig. 2b).

MICAL-1 regulates apoptosis through NDR kinases

Recently, a novel function for MICAL-1 in the regulation of apoptosis was described. In an unbiased protein-protein interaction screen, NDR1 and NDR2 kinases were identified as MICAL-1 interacting proteins [21]. NDR kinases belong to the serine/threonine AGC (protein kinase A (PKA)/PKG/PKC-like) kinase family and have important roles in cellular processes such as mitotic exit and apoptosis [62]. Ablation of NDR1 in mice predisposes to the development of cancer, presumably due to compromised apoptosis [63]. Like other AGC protein kinases, NDRs have a regulatory $\mathrm{C}$-terminal hydrophobic motif that is targeted by upstream MST kinases and required for kinase 
activity $[64,65]$. A series of expression, biochemical, and functional experiments showed that MICAL-1 binds the C-terminal hydrophobic motif of NDR1 and NDR2 and functions as an endogenous inhibitor of the MST (mammalian Ste20-related)-NDR pathway (Fig. 2c). MICAL-1 competes with MST1 for NDR binding and knockdown or overexpression of MICAL-1 in cells enhances or reduces NDR activity, respectively [21]. MST1-induced NDR1/2 activation plays a crucial role downstream of RASSF1A (Ras association domain family member 1A) in the apoptotic response to death receptor stimulation [65]. Interestingly, knockdown or overexpression of MICAL-1 in cells enhances or inhibits the response to pro-apoptotic stimuli such as etoposide and TNF- $\alpha$ [21], both capable of signaling through the MST-NDR pathway [63, 65]. Thus, MICAL-1 can negatively regulate the MST-NDR pathway thereby controlling pro-apoptotic signaling (Fig. 2c). Both genetically engineered mice and Drosophila models implicate NDR and MST kinases in apoptosis regulation in vivo [66-76]. Therefore, important future goals are to examine whether the MICAL/NDR/MST pathway also functions in invertebrate species and whether MICALdeficient mice or flies show altered apoptotic responses in vivo. This would require analysis of previously reported Mical-deficient flies and the generation and analysis of MICAL knockout mice.

It is possible that this MICAL-1-dependent regulatory mechanism also functions in MST- and/or NDR-dependent cellular processes other than apoptosis. For example, striking similarities exist between the effects of manipulating MST, MICAL, and NDR on neuronal morphology. Knockdown of MST3b or overexpression of constitutively active MICAL-1 in cultured mammalian neurons inhibits neurite growth [20, 77], while exogenous NDR2 expression enhances neurite growth [58]. Loss of Tricornered (NDR) or Hippo (MST) in Drosophila and of SAX-1 (NDR) in C. elegans leads to altered dendritic arborization and tiling defects (i.e., ectopic overlap between individual dendritic trees) [78-80]. Interestingly, loss of Drosophila Mical also results in enlarged dendritic fields due to deficits in dendritic pruning [14]. It is also interesting to note that knockdown of MST3b leads to a reduction in CRMP-1 [77], a putative substrate for the MICAL-1 MO domain. Thus, MICAL-1 may not only negatively regulate MST kinases, MSTs might also influence MICAL-1 function by controlling its substrate levels.

MICALs control several aspects of neural development

Thus far, MICAL proteins have been best characterized for their role during neural circuit development. As discussed in this section, MICALs contribute to the control of axon guidance, synaptic structure, dendritic pruning, and cell body positioning.

\section{Axon guidance}

One of the first MICAL proteins to be identified, Mical, is required for the guidance of embryonic axons in Drosophila by semaphorin proteins [3]. During the development of the nervous system, chemotactic molecules in the extracellular environment, known as axon guidance cues, instruct axons to follow molecularly predefined routes to their targets (Fig. 3). These guidance cues can either be diffusible or membrane-bound. Furthermore, they can prevent axons from growing into specific territories (i.e., function as axon repellents) or stimulate axon growth into a specific direction (i.e., axon attractants) [81]. Axon guidance cues are detected by cell surface receptors on the growth cone, a sensory structure at the tip of a growing axon. Binding of axon guidance cues to growth cone receptors triggers intracellular signaling events that ultimately control cytoskeletal dynamics [57].

Semaphorins form one of the five large families of canonical axon guidance cues and commonly use plexins as their receptors [82, 83]. Various co-receptors and intracellular effectors are involved in detecting and mediating the effects of semaphorins on axons [57, 84-86]. In

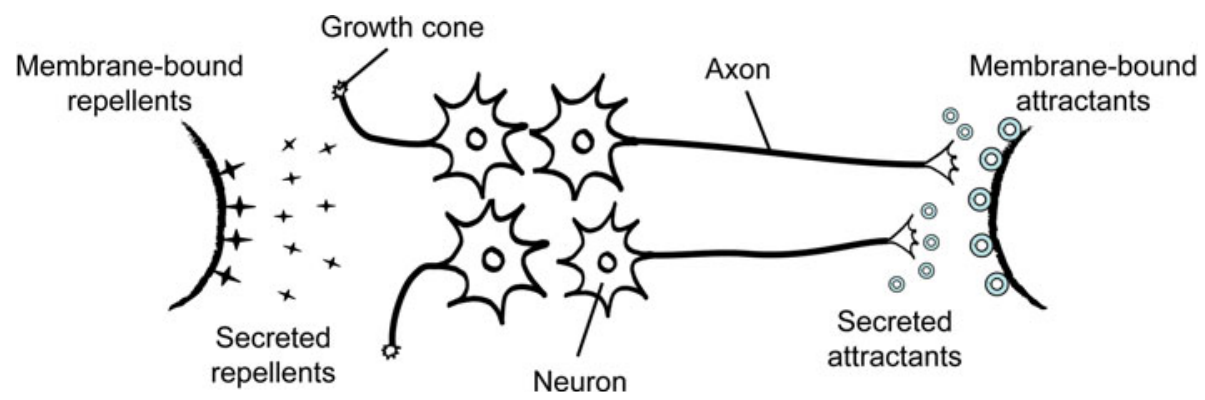

Fig. 3 Axon guidance. During development, neurons send out axons along molecularly predefined routes towards their synaptic targets. The growth cone at the tip of each axon senses environmental signals (guidance cues) that determine the direction of axon extension.
Diffusible or membrane-bound axon guidance cues can either prevent axons from growing into specific territories (i.e., act as axon repellents) or attract them into specific directions (axon attractants) 


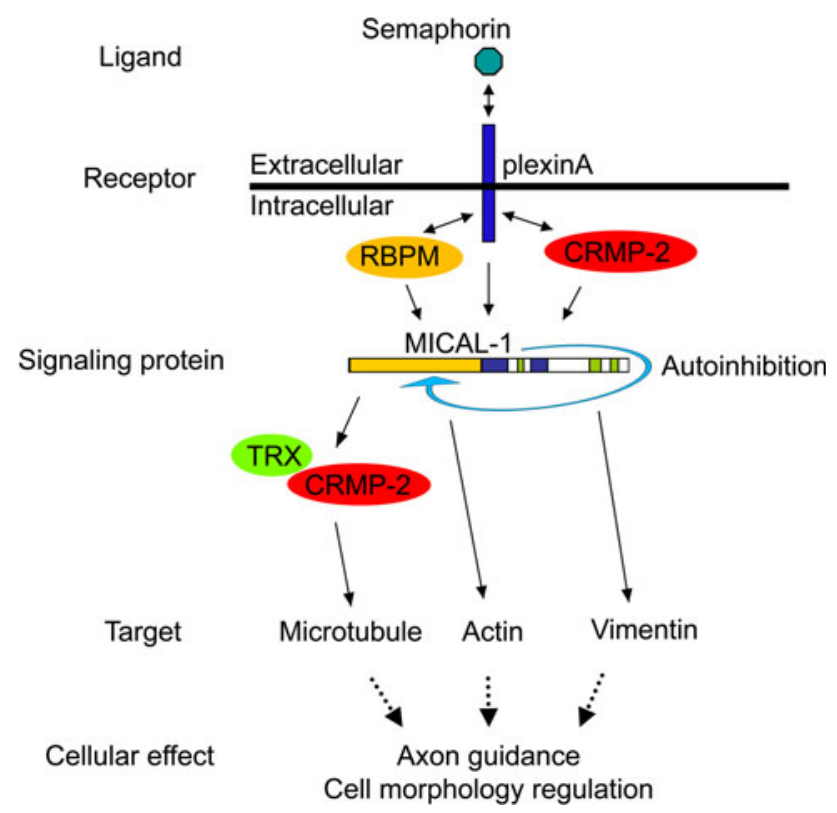

Fig. 4 MICALs in semaphorin/plexinA signaling. Semaphorins bind and activate plexinA receptors, thereby activating an intracellular signaling complex that contains the signaling proteins MICAL-1, CRMP (collapsin response mediator protein), and/or RanBPM (RBPM; Ran GTPase binding protein). CRMP and RanBPM bind MICAL-1 and plexinA receptors. Following semaphorin stimulation, MICAL-1 redox signaling is required for the interaction of CRMP-2 with TRX (thioredoxin), which primes CRMP-2 for phosphorylation by GSK-3 $\beta$ (glycogen synthase kinase- $3 \beta$ ) leading to microtubule disassembly and growth cone collapse. MICAL-1 is autoinhibited through intramolecular interactions and release of this inhibition activates MICAL-1 monooxygenase activity. Activation of plexinA has been proposed to release the autoinhibited conformation of MICAL-1. Mical, and presumably also other MICAL family members, can bind actin and induce disassembly of the actin cytoskeleton through its MO domain and redox signaling. Vimentin, an intermediate filament has been reported to bind MICAL-1. Thus, MICAL-1 may regulate different components of the cytoskeleton upon activation by plexinAs or other upstream signaling molecules

Drosophila, Mical is required for Sema-1a-PlexA-mediated repulsive axon guidance. Mical physically interacts with the PlexA cytosolic region and genetic inactivation of Mical disturbs the proper patterning of the (inter)segmental nerves that normally innervate the wing muscles. This guidance defect phenocopies abnormalities observed in Sema-1a and PlexA-deficient flies suggesting that Sema-1a, PlexA, and Mical function in the same pathway [3]. Given the ability of Mical to bind and modulate F-actin [15], it has been proposed that Mical forms a bridge between PlexA receptors and the cytoskeleton and induces cytoskeletal changes upon Sema-1a ligand binding.

Vertebrate MICALs have also been implicated in signaling downstream of semaphorins, i.e., class 3 and 6 semaphorins (Sema3 s and Sema6 s). Sema3 s are detected by a receptor complex composed of neuropilin and plexinA proteins. In this complex, neuropilins function as ligand- binding and plexinAs as signal-transducing subunits. Sema6 s bind and signal directly through plexinAs [57]. Although in vivo evidence for a role of MICALs in vertebrate axon guidance and plexinA signaling is lacking, several in vitro observations support a role for MICAL-1 in semaphorin/plexin signaling in axons (Fig. 4). First, MICAL-1 physically interacts with plexinA1 and plexinA3, and this interaction is enhanced by Sema3A stimulation [20]. Second, co-transfection of MICAL-1 and plexinA1 in COS-7 cells induces cell contraction resembling the contraction responses observed following Sema3A treatment of COS-7 cells exogenously expressing neuropilin1 and plexinA1 [20,87]. Third, MICAL-1 and $M I C A L-3$ knockdown reduces growth cone collapse responses induced by Sema3A [19]. Fourth, overexpression of MICAL-1 mutants lacking the MO domain in neurons abolishes the growth inhibitory effect of Sema3A on sensory axons [20]. Fifth, EGCG, a compound with the ability to block monooxygenases, neutralizes the axon repulsive effects of Sema3A and Sema3F [3, 5]. Sixth, MICAL-1 can bind several effectors of the Sema3A pathway including CRMPs and RanBPM (Ran small GTPase binding protein) $[55,88]$. CRMPs can associate with tubulin heterodimers and promote microtubule assembly [54, 55]. MICAL-1 binds CRMP-2, and other CRMP family members [20], and is required for the oxidation-dependent phosphorylation and inactivation of CRMP- 2 by GSK-3 $\beta$ (glycogen synthase kinase-3 $\beta$ ) leading to microtubule disassembly (Fig. 2a) [19].

Synaptic structure and dendritic pruning

In a postembryonic screen designed to identify proteins that control the maintenance and remodeling of neuromuscular synapses in Drosophila, Mical was identified as a critical regulator of synaptic structure [13]. Mical is essential for both synaptic bouton distribution along muscle fibers and for the organization of the postsynaptic domain. In Mical mutant flies, synaptic boutons cluster around initial nerve-muscle contact sites and along synaptic branches and fail to properly spread along the muscle fiber. In addition, deranged actin and myosin filaments accumulate in muscle cells in Mical mutants and interfere with the organization of the postsynaptic region [13]. As a result, postsynaptic structures are deeply embedded in the muscle fiber. The presence of MICAL proteins in adult mouse synaptosomes [89] supports the idea that MICALs may also subserve functions at vertebrate synapses.

In addition to axon guidance and synaptic structure, Mical contributes to dendritic pruning in Drosophila [14]. Dendritic pruning is an important refinement process that selectively removes exuberant or inaccurate neuronal processes during development without causing neuronal cell 
death [90]. In Drosophila, larval-born neurons undergo extensive remodeling at the early metamorphosis stage to construct adult neuronal connections. During this stage, Mical expression is upregulated by Sox 14, an ecdysoneinduced transcription factor. Mical depletion in Drosophila results in a failure to severe inappropriate dendrites from the neuronal cell body leading to exuberant dendritic trees [14]. The initial steps of dendrite severing involve blebbing and proximal thinning of dendrites mediated by the depolymerization of the microtubule and actin cytoskeletons. Although further work is needed to establish the precise role of Mical during dendritic pruning, it is tempting to speculate that Mical controls the severing of dendrites through the depolymerization of actin.

\section{Cell body positioning}

In the chick spinal cord, MICAL-3 controls the proper positioning of motor neuron somata [17]. Spinal motor neuron cell bodies reside in the CNS whereas their axon projections exit the CNS to contact targets in the periphery, i.e., muscle cells. During spinal cord development, the cell bodies of motor neurons migrate along their motor axons to reach their proper positions in the spinal cord, a process called somal translocation. A population of neural crestderived cells, boundary cap cells, is located at the border of the CNS and PNS and prevents motor neuron cell bodies from exiting the CNS along motor axons [91, 92]. Genetic studies in chick indicate that Sema6A expressed on boundary cap cells repels motor axons expressing plexinA2. Knockdown of Sema6A and plexinA2 causes motor neurons to ectopically migrate outside the spinal cord into the ventral root. Knockdown of MICAL-3 induces a similar phenotype hinting at the existence of a Sema/plexinA2/ MICAL-3 pathway during motor neuron cell body positioning [17].

\section{Concluding remarks and future perspectives}

Our knowledge of the cellular effects of MICALs has advanced significantly over the past several years, and the molecular processes that underlie these effects are being unveiled at a rapid pace. A major breakthrough in defining MICAL function is the demonstration that Mical can bind to and modify F-actin through redox reactions [15]. Although the ability to regulate actin dynamics remains to be shown for MICALs other than Drosophila Mical, redox regulation of the cytoskeleton is considered a common feature of MICAL family members. Many of the cellular functions of MICALs reported so far are thought to rely on their ability to directly modify the cytoskeleton, e.g., regulation of axon guidance, dendritic pruning, and synaptic bouton distribution. In addition to F-actin, it will be interesting to determine whether MICALs can redox modify other components of the cytoskeleton, e.g., vimentin, and whether such events rely on intermediates such as $\mathrm{H}_{2} \mathrm{O}_{2}$ or on direct redox modification by the MICAL MO domain. Recent work shows that exocytosis of Rab8-positive vesicles also requires an intact MICAL MO domain [18]. However, during this process MICAL-3 does not appear to regulate the cytoskeleton but rather its own turnover through the MO domain. How the MO domain can regulate protein turnover and whether such a mechanism is also important for other reported cellular functions of MICALs remains to be determined.

In contrast to cytoskeletal regulation and exocytosis, a recently reported function of MICAL-1 in apoptosis regulation does not depend on the MO domain. Instead, the MICAL-1 LIM and C-terminal regions bind NDR kinases, thereby inhibiting binding and activation by upstream MST kinases [21]. The factors that regulate MICAL activity in the MST-NDR pathway, as well as in other pathways, remain unknown. However, the recent identification of MICALs as phosphoproteins suggests that protein kinases may be upstream regulators of MICAL function. Furthermore, proteins that antagonize the autoinhibited conformation of MICAL proteins may function to activate these proteins. Although MICALs contain several domains and motifs capable of interacting with other proteins, the binding partners of most of these regions remain unknown. Identification of the full complement of MICAL binding partners will provide valuable insight in the precise substrates for different MICAL proteins, regulators of their activity and unknown biological pathways.

Several studies using Drosophila embryos deficient in Mical or overexpressing truncated or full-length Mical genes have firmly established the role of Mical in cellular processes such as axon guidance and bristle formation. Future studies are needed, however, to examine whether vertebrate MICAL proteins subserve similar in vivo functions. Since MICAL-1, MICAL-2 and MICAL-3 display highly overlapping patterns of expression (e.g., [5]) this may require the simultaneous inactivation of multiple $M I C A L$ genes in mice in vivo.

Recent studies implicate MICAL proteins in spinal cord injury and cancer. Following spinal cord injury, MICAL expression is regulated in cells associated with the glial scar and the neurite growth inhibitory environment of the injured CNS [5]. In addition, splicing variants of $M I C A L-2$ (MICAL2-PVs) are associated with prostate cancer progression. Intriguingly, knockdown of MICAL2-PVs in prostate cancer greatly reduces cancer cell viability [16] Therefore, we can expect that experiments over the next years will not only advance our understanding of MICAL 
function and signaling but also will lead to therapeutic advances in injury and disease.

Acknowledgments Work in the laboratory of the authors on MICALs was supported by the Netherlands Organization for Health Research and Development (ZonMW-VIDI and ZonMW-TOP), the Human Frontier Science Program (HFSP-CDA), and the Genomics Center Utrecht (to R.J.P.).

Open Access This article is distributed under the terms of the Creative Commons Attribution Noncommercial License which permits any noncommercial use, distribution, and reproduction in any medium, provided the original author(s) and source are credited.

\section{References}

1. Kolk SM, Pasterkamp RJ (2007) MICAL flavoprotein monooxygenases: structure, function and role in semaphorin signaling. Adv Exp Med Biol 600:38-51. doi:10.1007/978-0-387-70956-7

2. Suzuki T, Nakamoto T, Ogawa S, Seo S, Matsumura T, Tachibana K, Morimoto C, Hirai H (2002) MICAL, a novel CasL interacting molecule, associates with vimentin. J Biol Chem 277(17):14933-14941. doi:10.1074/jbc.M111842200

3. Terman JR, Mao T, Pasterkamp RJ, Yu HH, Kolodkin AL (2002) MICALs, a family of conserved flavoprotein oxidoreductases, function in plexin-mediated axonal repulsion. Cell 109(7):887900 S0092867402007948[pii]

4. Fischer J, Weide T, Barnekow A (2005) The MICAL proteins and Rab1: a possible link to the cytoskeleton? Biochem Biophys Res Commun 328(2):415-423. doi:10.1016/j.bbrc.2004.12.182

5. Pasterkamp RJ, Dai HN, Terman JR, Wahlin KJ, Kim B, Bregman BS, Popovich PG, Kolodkin AL (2006) MICAL flavoprotein monooxygenases: expression during neural development and following spinal cord injuries in the rat. Mol Cell Neurosci 31(1):52-69. doi:10.1016/j.men.2005.09.001

6. Weide T, Teuber J, Bayer M, Barnekow A (2003) MICAL-1 isoforms, novel Rab1-interacting proteins. Biochem Biophys Res Commun 306(1):79-86 S0006291X03009185[pii]

7. Xue Y, Kuok C, Xiao A, Zhu Z, Lin S, Zhang B (2010) Identification and expression analysis of mical family genes in zebrafish. J Genet Genomics 37(10):685-693. doi:10.1016/ S1673-8527(09)60086-2

8. Terai T, Nishimura N, Kanda I, Yasui N, Sasaki T (2006) JRAB/ MICAL-L2 is a junctional Rab13-binding protein mediating the endocytic recycling of occludin. Mol Biol Cell 17(5):2465-2475. doi:10.1091/mbc.E05-09-0826

9. Nishimura N, Sasaki T (2008) Identification and characterization of JRAB/MICAL-L2, a junctional Rab13-binding protein. Methods Enzymol 438:141-153. doi:10.1016/S0076-6879 (07)38010-5

10. Nishimura N, Sasaki T (2009) Rab family small G proteins in regulation of epithelial apical junctions. Front Biosci 14:2115-2129 3366[pii]

11. Sharma M, Giridharan SS, Rahajeng J, Caplan S, Naslavsky N (2010) MICAL-L1: an unusual Rab effector that links EHD1 to tubular recycling endosomes. Commun Integr Biol 3(2):181-183

12. Rahajeng J, Giridharan SS, Cai B, Naslavsky N, Caplan S (2010) Important relationships between Rab and MICAL proteins in endocytic trafficking. World J Biol Chem 1(8):254-264. doi: 10.4331/wjbc.v1.i8.254

13. Beuchle D, Schwarz H, Langegger M, Koch I, Aberle H (2007) Drosophila MICAL regulates myofilament organization and synaptic structure. Mech Dev 124(5):390-406. doi:10.1016/ j.mod.2007.01.006

14. Kirilly D, Gu Y, Huang Y, Wu Z, Bashirullah A, Low BC, Kolodkin AL, Wang H, Yu F (2009) A genetic pathway composed of Sox14 and Mical governs severing of dendrites during pruning. Nat Neurosci 12(12):1497-1505. doi:10.1038/nn.2415

15. Hung RJ, Yazdani U, Yoon J, Wu H, Yang T, Gupta N, Huang Z, van Berkel WJ, Terman JR (2010) Mical links semaphorins to F-actin disassembly. Nature 463(7282):823-827. doi:10.1038/ nature 08724

16. Ashida S, Furihata M, Katagiri T, Tamura K, Anazawa Y, Yoshioka H, Miki T, Fujioka T, Shuin T, Nakamura Y, Nakagawa H (2006) Expression of novel molecules, MICAL2-PV (MICAL2 prostate cancer variants), increases with high Gleason score and prostate cancer progression. Clin Cancer Res 12(9):2767-2773. doi:10.1158/1078-0432.CCR-05-1995

17. Bron R, Vermeren M, Kokot N, Andrews W, Little GE, Mitchell KJ, Cohen J (2007) Boundary cap cells constrain spinal motor neuron somal migration at motor exit points by a semaphorinplexin mechanism. Neural Dev 2:21. doi:10.1186/17498104-2-21

18. Grigoriev I, Yu KL, Martinez-Sanchez E, Serra-Marques A, Smal I, Meijering E, Demmers J, Peranen J, Pasterkamp RJ, van der Sluijs P, Hoogenraad CC, Akhmanova A (2011) Rab6, Rab8, and MICAL3 cooperate in controlling docking and fusion of exocytotic carriers. Curr Biol 21(11):967-974. doi:10.1016/j.cub. 2011.04.030

19. Morinaka A, Yamada M, Itofusa R, Funato Y, Yoshimura Y, Nakamura F, Yoshimura T, Kaibuchi K, Goshima Y, Hoshino M, Kamiguchi H, Miki H (2011) Thioredoxin mediates oxidationdependent phosphorylation of crmp2 and growth cone collapse. Sci Signal 4(170):ra26. doi:10.1126/scisignal.2001127

20. Schmidt EF, Shim SO, Strittmatter SM (2008) Release of MICAL autoinhibition by semaphorin-plexin signaling promotes interaction with collapsin response mediator protein. J Neurosci 28(9):2287-2297. doi:10.1523/JNEUROSCI.5646-07.2008

21. Zhou Y, Adolfs Y, Pijnappel WMMP, Fuller SJ, Van der Schors RC, Li KW, Sugden PH, Smit AB, Hergovich A, Pasterkamp RJ (2011) MICAL-1 is a negative regulator of MST-NDR kinase signaling and apoptosis. Mol Cell Biol. doi:10.1128/MCB. 01389-10

22. Nadella M, Bianchet MA, Gabelli SB, Barrila J, Amzel LM (2005) Structure and activity of the axon guidance protein MICAL. Proc Natl Acad Sci USA 102(46):16830-16835. doi: 10.1073/pnas.0504838102

23. Siebold C, Berrow N, Walter TS, Harlos K, Owens RJ, Stuart DI, Terman JR, Kolodkin AL, Pasterkamp RJ, Jones EY (2005) High-resolution structure of the catalytic region of MICAL (molecule interacting with CasL), a multidomain flavoenzymesignaling molecule. Proc Natl Acad Sci USA 102(46):1683616841. doi:10.1073/pnas.0504997102

24. Cole LJ, Entsch B, Ortiz-Maldonado M, Ballou DP (2005) Properties of $p$-hydroxybenzoate hydroxylase when stabilized in its open conformation. Biochemistry 44(45):14807-14817. doi: 10.1021/bi0512142

25. Entsch B, Cole LJ, Ballou DP (2005) Protein dynamics and electrostatics in the function of $p$-hydroxybenzoate hydroxylase. Arch Biochem Biophys 433(1):297-311. doi:10.1016/j.abb. 2004.09.029

26. Abe I, Kashiwagi K, Noguchi H (2000) Antioxidative galloyl esters as enzyme inhibitors of $p$-hydroxybenzoate hydroxylase. FEBS Lett 483(2-3):131-134 S0014-5793(00)02100-1[pii]

27. Abe I, Seki T, Umehara K, Miyase T, Noguchi H, Sakakibara J, Ono T (2000) Green tea polyphenols: novel and potent inhibitors of squalene epoxidase. Biochem Biophys Res Commun 268(3):767-771. doi:10.1006/bbrc.2000.2217 
28. Rozenblum GT, Gimona M (2008) Calponins: adaptable modular regulators of the actin cytoskeleton. Int $\mathrm{J}$ Biochem Cell Biol 40(10):1990-1995. doi:10.1016/j.biocel.2007.07.010

29. Gimona M, Djinovic-Carugo K, Kranewitter WJ, Winder SJ (2002) Functional plasticity of $\mathrm{CH}$ domains. FEBS Lett 513(1):98-106 S0014579301032409[pii]

30. Fraley TS, Pereira CB, Tran TC, Singleton C, Greenwood JA (2005) Phosphoinositide binding regulates alpha-actinin dynamics: mechanism for modulating cytoskeletal remodeling. J Biol Chem 280(15):15479-15482. doi:10.1074/jbc.M500631200

31. Fukami K, Furuhashi K, Inagaki M, Endo T, Hatano S, Takenawa $\mathrm{T}$ (1992) Requirement of phosphatidylinositol 4, 5-bisphosphate for alpha-actinin function. Nature 359(6391):150-152. doi: $10.1038 / 359150 \mathrm{a} 0$

32. Korenbaum E, Rivero F (2002) Calponin homology domains at a glance. J Cell Sci 115(Pt 18):3543-3545

33. Sun H, Dai H, Zhang J, Jin X, Xiong S, Xu J, Wu J, Shi Y (2006) Solution structure of calponin homology domain of Human MICAL-1. J Biomol NMR 36(4):295-300. doi:10.1007/s10858006-9062-5

34. Bach I (2000) The LIM domain: regulation by association. Mech Dev 91(1-2):5-17 S0925-4773(99)00314-7[pii]

35. Dawid IB, Breen JJ, Toyama R (1998) LIM domains: multiple roles as adapters and functional modifiers in protein interactions. Trends Genet 14(4):156-162 S0168-9525(98)01424-3[pii]

36. Kadrmas JL, Beckerle MC (2004) The LIM domain: from the cytoskeleton to the nucleus. Nat Rev Mol Cell Biol 5(11):920931. doi:10.1038/nrm1499

37. Zheng Q, Zhao Y (2007) The diverse biofunctions of LIM domain proteins: determined by subcellular localization and protein-protein interaction. Biol Cell 99(9):489-502. doi: 10.1042/BC20060126

38. Williamson MP (1994) The structure and function of proline-rich regions in proteins. Biochem J 297(Pt 2):249-260

39. Kay BK, Williamson MP, Sudol M (2000) The importance of being proline: the interaction of proline-rich motifs in signaling proteins with their cognate domains. FASEB J 14(2):231-241

40. Rayala SK, den Hollander P, Manavathi B, Talukder AH, Song C, Peng S, Barnekow A, Kremerskothen J, Kumar R (2006) Essential role of KIBRA in co-activator function of dynein light chain 1 in mammalian cells. J Biol Chem 281(28):19092-19099. doi:10.1074/jbc.M600021200

41. Woodcock CL, Dimitrov S (2001) Higher-order structure of chromatin and chromosomes. Curr Opin Genet Dev 11(2):130-135 S0959-437X(00)00169-6[pii]

42. Vadlamudi RK, Wang RA, Mazumdar A, Kim Y, Shin J, Sahin A, Kumar R (2001) Molecular cloning and characterization of PELP1, a novel human coregulator of estrogen receptor alpha. J Biol Chem 276(41):38272-38279. doi:10.1074/jbc.M1037832

43. Wolf E, Kim PS, Berger B (1997) MultiCoil: a program for predicting two- and three-stranded coiled coils. Protein Sci 6(6):1179-1189. doi:10.1002/pro.5560060606

44. Burkhard P, Stetefeld J, Strelkov SV (2001) Coiled coils: a highly versatile protein folding motif. Trends Cell Biol 11(2):82-88 S0962-8924(00)01898-5[pii]

45. Rikova K, Guo A, Zeng Q, Possemato A, Yu J, Haack H, Nardone J, Lee K, Reeves C, Li Y, Hu Y, Tan Z, Stokes M, Sullivan L, Mitchell J, Wetzel R, Macneill J, Ren JM, Yuan J, Bakalarski CE, Villen J, Kornhauser JM, Smith B, Li D, Zhou X, Gygi SP, Gu TL, Polakiewicz RD, Rush J, Comb MJ (2007) Global survey of phosphotyrosine signaling identifies oncogenic kinases in lung cancer. Cell 131(6):1190-1203. doi:10.1016/j.cell.2007.11.025

46. Dephoure N, Zhou C, Villen J, Beausoleil SA, Bakalarski CE, Elledge SJ, Gygi SP (2008) A quantitative atlas of mitotic phosphorylation. Proc Natl Acad Sci U S A 105(31):1076210767. doi:10.1073/pnas.0805139105
47. Tilney LG, DeRosier DJ (2005) How to make a curved Drosophila bristle using straight actin bundles. Proc Natl Acad Sci USA 102(52):18785-18792. doi:10.1073/pnas.0509437102

48. Dalle-Donne I, Rossi R, Milzani A, Di Simplicio P, Colombo R (2001) The actin cytoskeleton response to oxidants: from small heat shock protein phosphorylation to changes in the redox state of actin itself. Free Radic Biol Med 31(12):1624-1632 S0891584901007493[pii]

49. Dalle-Donne I, Rossi R, Giustarini D, Gagliano N, Lusini L, Milzani A, Di Simplicio P, Colombo R (2001) Actin carbonylation: from a simple marker of protein oxidation to relevant signs of severe functional impairment. Free Radic Biol Med 31(9):1075-1083 S0891584901006906[pii]

50. Milzani A, DalleDonne I, Colombo R (1997) Prolonged oxidative stress on actin. Arch Biochem Biophys 339(2):267-274. doi: 10.1006/abbi.1996.9847

51. Rogers KR, Morris CJ, Blake DR (1991) Oxidation of thiol in the vimentin cytoskeleton. Biochem J 275(Pt 3):789-791

52. Landino LM, Moynihan KL, Todd JV, Kennett KL (2004) Modulation of the redox state of tubulin by the glutathione/ glutaredoxin reductase system. Biochem Biophys Res Commun 314(2):555-560 S0006291X03027529[pii]

53. Bouton AH, Riggins RB, Bruce-Staskal PJ (2001) Functions of the adapter protein Cas: signal convergence and the determination of cellular responses. Oncogene 20(44):6448-6458. doi: 10.1038/sj.onc. 1204785

54. Fukata Y, Itoh TJ, Kimura T, Menager C, Nishimura T, Shiromizu $\mathrm{T}$, Watanabe $\mathrm{H}$, Inagaki $\mathrm{N}$, Iwamatsu A, Hotani $\mathrm{H}$, Kaibuchi K (2002) CRMP-2 binds to tubulin heterodimers to promote microtubule assembly. Nat Cell Biol 4(8):583-591. doi: $10.1038 / \mathrm{nc}$

55. Schmidt EF, Strittmatter SM (2007) The CRMP family of proteins and their role in Sema3A signaling. Adv Exp Med Biol 600:1-11. doi:10.1007/978-0-387-70956-7

56. Ahmed A, Eickholt BJ (2007) Intracellular kinases in semaphorin signaling. Adv Exp Med Biol 600:24-37. doi:10.1007/978-0-38770956-7

57. Zhou Y, Gunput RA, Pasterkamp RJ (2008) Semaphorin signaling: progress made and promises ahead. Trends Biochem Sci 33(4):161-170. doi:10.1016/j.tibs.2008.01.006

58. Stork O, Zhdanov A, Kudersky A, Yoshikawa T, Obata K, Pape HC (2004) Neuronal functions of the novel serine/threonine kinase Ndr2. J Biol Chem 279(44):45773-45781. doi:10.1074/ jbc.M40

59. Grosshans BL, Ortiz D, Novick P (2006) Rabs and their effectors: achieving specificity in membrane traffic. Proc Natl Acad Sci USA 103(32):11821-11827. doi:10.1073/pnas.0601617103

60. Fukuda M, Kanno E, Ishibashi K, Itoh T (2008) Large-scale screening for novel Rab effectors reveals unexpected broad Rab binding specificity. Mol Cell Proteomics 7(6):1031-1042. doi: 10.1074/mcp.M700569-MCP200

61. Yamamura R, Nishimura N, Nakatsuji H, Arase S, Sasaki T (2008) The interaction of JRAB/MICAL-L2 with Rab8 and Rab13 coordinates the assembly of tight junctions and adherens junctions. Mol Biol Cell 19(3):971-983. doi:10.1091/mbc.E0706-0551

62. Hergovich A, Stegert MR, Schmitz D, Hemmings BA (2006) NDR kinases regulate essential cell processes from yeast to humans. Nat Rev Mol Cell Biol 7(4):253-264. doi:10.1038/ nrm1891

63. Cornils H, Stegert MR, Hergovich A, Hynx D, Schmitz D, Dirnhofer S, Hemmings BA (2010) Ablation of the kinase NDR1 predisposes mice to the development of $\mathrm{T}$ cell lymphoma. Sci Signal 3(126):ra47. doi:10.1126/scisignal.2000681

64. Stegert MR, Hergovich A, Tamaskovic R, Bichsel SJ, Hemmings BA (2005) Regulation of NDR protein kinase by hydrophobic 
motif phosphorylation mediated by the mammalian Ste20-like kinase MST3. Mol Cell Biol 25(24):11019-11029. doi: 10.1128/MCB.25.24.11019-11029.2005

65. Vichalkovski A, Gresko E, Cornils H, Hergovich A, Schmitz D, Hemmings BA (2008) NDR kinase is activated by RASSF1A/ MST1 in response to Fas receptor stimulation and promotes apoptosis. Curr Biol 18(23):1889-1895. doi:10.1016/j.cub.2008. 10.060

66. Choi J, Oh S, Lee D, Oh HJ, Park JY, Lee SB, Lim DS (2009) Mst1-FoxO signaling protects Naive T lymphocytes from cellular oxidative stress in mice. PLoS One 4(11):e8011. doi:10.1371/ journal.pone.0008011

67. Harvey KF, Pfleger CM, Hariharan IK (2003) The Drosophila Mst ortholog, hippo, restricts growth and cell proliferation and promotes apoptosis. Cell 114(4):457-467 S009286740300 5579 [pii]

68. Jia J, Zhang W, Wang B, Trinko R, Jiang J (2003) The Drosophila Ste20 family kinase dMST functions as a tumor suppressor by restricting cell proliferation and promoting apoptosis. Genes Dev 17(20):2514-2519. doi:10.1101/gad.1134003

69. Katagiri K, Katakai T, Ebisuno Y, Ueda Y, Okada T, Kinashi T (2009) Mst1 controls lymphocyte trafficking and interstitial motility within lymph nodes. EMBO J 28(9):1319-1331. doi: 10.1038/emboj.2009.82

70. Lu L, Li Y, Kim SM, Bossuyt W, Liu P, Qiu Q, Wang Y, Halder G, Finegold MJ, Lee JS, Johnson RL (2010) Hippo signaling is a potent in vivo growth and tumor suppressor pathway in the mammalian liver. Proc Natl Acad Sci USA 107(4):1437-1442. doi:10.1073/pnas.0911427107

71. Oh S, Lee D, Kim T, Kim TS, Oh HJ, Hwang CY, Kong YY, Kwon KS, Lim DS (2009) Crucial role for Mst1 and Mst2 kinases in early embryonic development of the mouse. Mol Cell Biol 29(23):6309-6320. doi:10.1128/MCB.00551-09

72. Pantalacci S, Tapon N, Leopold P (2003) The Salvador partner Hippo promotes apoptosis and cell-cycle exit in Drosophila. Nat Cell Biol 5(10):921-927. doi:10.1038/ncb1051

73. Song H, Mak KK, Topol L, Yun K, Hu J, Garrett L, Chen Y, Park O, Chang J, Simpson RM, Wang CY, Gao B, Jiang J, Yang Y (2010) Mammalian Mst1 and Mst2 kinases play essential roles in organ size control and tumor suppression. Proc Natl Acad Sci USA 107(4):1431-1436. doi:10.1073/pnas.0911409107

74. Udan RS, Kango-Singh M, Nolo R, Tao C, Halder G (2003) Hippo promotes proliferation arrest and apoptosis in the Salvador/Warts pathway. Nat Cell Biol 5(10):914-920. doi: 10.1038/ncb1050ncb1050[pii]

75. Zhou D, Conrad C, Xia F, Park JS, Payer B, Yin Y, Lauwers GY, Thasler W, Lee JT, Avruch J, Bardeesy N (2009) Mst1 and Mst2 maintain hepatocyte quiescence and suppress hepatocellular carcinoma development through inactivation of the Yap1 oncogene. Cancer Cell 16(5):425-438. doi:10.1016/j.ccr.2009.09.026

76. Zhou D, Medoff BD, Chen L, Li L, Zhang XF, Praskova M, Liu M, Landry A, Blumberg RS, Boussiotis VA, Xavier R, Avruch J (2008) The Nore1B/Mst1 complex restrains antigen receptorinduced proliferation of naive T cells. Proc Natl Acad Sci USA 105(51):20321-20326. doi:10.1073/pnas.0810773105
77. Irwin N, Li YM, O’Toole JE, Benowitz LI (2006) Mst3b, a purine-sensitive Ste20-like protein kinase, regulates axon outgrowth. Proc Natl Acad Sci USA 103(48):18320-18325. doi: 10.1073/pnas.0605135103

78. Emoto K, He Y, Ye B, Grueber WB, Adler PN, Jan LY, Jan YN (2004) Control of dendritic branching and tiling by the Tricornered-kinase/Furry signaling pathway in Drosophila sensory neurons. Cell 119(2):245-256. doi:10.1016/j.cell.2004.09.036

79. Emoto K, Parrish JZ, Jan LY, Jan YN (2006) The tumour suppressor Hippo acts with the NDR kinases in dendritic tiling and maintenance. Nature 443(7108):210-213. doi:10.1038/nature 05090

80. Gallegos ME, Bargmann CI (2004) Mechanosensory neurite termination and tiling depend on SAX-2 and the SAX-1 kinase. Neuron 44(2):239-249. doi:10.1016/j.neuron.2004.09.021

81. Tessier-Lavigne M, Goodman CS (1996) The molecular biology of axon guidance. Science 274(5290):1123-1133

82. Tran TS, Kolodkin AL, Bharadwaj R (2007) Semaphorin regulation of cellular morphology. Annu Rev Cell Dev Biol 23:263-292. doi:10.1146/annurev.cellbio.22.010605.093554

83. Pasterkamp RJ, Giger RJ (2009) Semaphorin function in neural plasticity and disease. Curr Opin Neurobiol 19(3):263-274. doi: 10.1016/j.conb.2009.06.001

84. Derijck AA, Van Erp S, Pasterkamp RJ (2010) Semaphorin signaling: molecular switches at the midline. Trends Cell Biol 20(9):568-576. doi:10.1016/j.tcb.2010.06.007

85. Franco M, Tamagnone L (2008) Tyrosine phosphorylation in semaphorin signalling: shifting into overdrive. EMBO Rep 9(9):865-871. doi:10.1038/embor.2008.139

86. Jackson RE, Eickholt BJ (2009) Semaphorin signalling. Curr Biol 19(13):R504-R507. doi:10.1016/j.cub.2009.04.055

87. Takahashi T, Fournier A, Nakamura F, Wang LH, Murakami Y, Kalb RG, Fujisawa H, Strittmatter SM (1999) Plexin-neuropilin-1 complexes form functional semaphorin-3A receptors. Cell 99(1):59-69 S0092-8674(00)80062-8[pii]

88. Togashi H, Schmidt EF, Strittmatter SM (2006) RanBPM contributes to Semaphorin3A signaling through plexin-A receptors. J Neurosci 26(18):4961-4969. doi:10.1523/JNEUROSCI.070406.2006

89. Dahlhaus M, Wan Li K, van der Schors RC, Saiepour MH, van Nierop P, Heimel JA, Hermans JM, Loos M, Smit AB, Levelt CN (2011) The synaptic proteome during development and plasticity of the mouse visual cortex. Mol Cell Proteomics 10(5):M110 005413. doi:10.1074/mcp.M110.005413

90. Luo L, O'Leary DD (2005) Axon retraction and degeneration in development and disease. Annu Rev Neurosci 28:127-156. doi: 10.1146/annurev.neuro.28.061604.135632

91. Schmidt ER, Pasterkamp RJ, van den Berg LH (2009) Axon guidance proteins: novel therapeutic targets for ALS? Prog Neurobiol 88(4):286-301. doi:S0301-0082(09)00077-X[pii] 10.1016/j.pneurobio.2009.05.004

92. Chauvet S, Rougon G (2008) Semaphorins deployed to repel cell migrants at spinal cord borders. J Biol 7(2):4. doi:10.1186/jbiol65 